\section{BJOS

Volume 17

2018

e181097

\title{
Photoelastic and finite element stress analysis reliability for implant-supported system stress investigation
}

Anna Gabriella Camacho Presotto ${ }^{1}$, Cláudia Lopes Brilhante Bhering ${ }^{2}$, Ricardo Armini Caldas ${ }^{1}$, Rafael Leonardo Xediek Consani', Valentim Adelino Ricardo Barão', Marcelo Ferraz Mesquita'

'Department of Prosthodontics and Periodontology, Piracicaba Dental School, University of Campinas (UNICAMP), Piracicaba, Sao Paulo, Brazil

2 Department of Restorative Dentistry, School of Dentistry, Federal University of Minas Gerais (UFMG), Minas Gerais, Brazil
Corresponding author: Anna Gabriella Camacho Presotto Department of Prosthodontics and Periodontology

University of Campinas (UNICAMP) Piracicaba Dental School Av Limeira, 901, Piracicaba São Paulo, 13414-903, Brazil. Tel. + +55-19 21065719 E-mail: annapresotto@gmail.com

Received: May 22, 2018 Accepted: July 09, 2018
Aim: To compare the reliability between photoelastic and finite element (FE) analyses by evaluating the effect of different marginal misfit levels on the stresses generated on two different implant-supported systems using conventional and short implants. Methods: Two photoelastic models were obtained: model $\mathrm{C}$ with two conventional implants $(4.1 \times 11 \mathrm{~mm})$; and model $S$ with a conventional and a short implant $(5 \times 6 \mathrm{~mm})$. Three-unit $\mathrm{CoCr}$ frameworks were fabricated simulating a superior first pre-molar (P) to first molar (M) fixed dental prosthesis. Different levels of misfit $(\mu \mathrm{m})$ were selected based on the misfit average of 10 frameworks obtained by the single-screw test protocol: low $(<20)$, medium $(>20$ and $<40)$ and high $(>40)$. Stress levels and distribution were measured by photoelastic analysis. A similar situation of the in vitro assay was designed and simulated by the in silico analysis. Maximum and minimum principal strain were recorded numerically and color-coded for the models. Von Mises Stress was obtained for the metallic components. Results: Photoelasticity and FE analyses showed similar tendency where the increase of misfit generates higher stress levels despite of the implant design. The short implant showed lower von Mises stress values; however, it presented stresses around its full length for the in vitro and in silico analysis. Also, model S showed higher $\mu$ strain values for all simulated misfit levels. The type of implant did not affect the stresses around pillar P. Conclusions: Photoelasticity and FEA are reliable methodologies presenting similarity for the investigation of the biomechanical behavior of implant-supported rehabilitations.

Keywords: Biomechanical Phenomena. Dental Implants Optical Phenomena. Finite Element Analysis. 


\section{Introduction}

Despite the high success rates of implant-supported rehabilitation demonstrated over the years, ${ }^{1}$ this issue continues to be an ongoing research goal in Dentistry. The attention in this field persists as clinical problems may occur with implants due to the considerable difference in the biomechanical behavior of implants and natural teeth. ${ }^{2}$ Differently from natural teeth, bone tissue and implants are rigidly connected and do not allow stress absorption as promoted by the presence of periodontal ligament. ${ }^{3}$ As a consequence, misfit can generate stresses in the implant system even without load application. ${ }^{34}$ Since the presence of misfit is a clinical reality, obtaining acceptable fit levels is very important to the longevity of the implant treatment, ${ }^{5,6}$ otherwise biological and mechanical complications can potentially occur. ${ }^{7}$

However, not only the presence of misfit affects the stresses in the implant-supported system, but also the selected components such as implant types and prosthetic abutments. ${ }^{8}$ In this sense, short and wide implants reduce the stress and strain in the periimplant bone in the posterior maxilla in comparison to conventional implants placed in the grafted sinus. ${ }^{9,10}$ Also, short implants $(<10 \mathrm{~mm})$ can avoid surgical procedures such as ridge augmentation and sinus lift, which involves greater morbidity for the patient, longer treatments and higher costs. ${ }^{8-10}$

Short implants can be a predictable alternative when used under rigorous clinical protocols, ${ }^{11}$ such as the optimization of occlusion of the prostheses, ${ }^{8}$ where its deficiency is a common cause of implant-supported rehabilitation failure..$^{12}$ In addition, the increase in diameter of short implants shows benefit for stress distribution. ${ }^{13} \mathrm{~A}$ previous review article evaluating the biomechanical behavior of short implants showed a similar survival rate and bone resorption when compared to the conventional implants. ${ }^{8}$ However, despite the decrease of the stress values around the implants, ${ }^{9,10}$ the influence of marginal misfit in this type of implants was not evaluated.

In vitro investigations of stress levels can be considered a challenge in prosthodontics researches as each methodology has its own singularities. Among the main methods used for in vitro stress investigation are photoelasticity2.4 and finite element analysis (FEA). ${ }^{2.1415}$ Photoelasticity provides a qualitative result of the stress distribution and mimics the clinical scenario by using real components (i.e. implants, abutments and frameworks). ${ }^{24,16}$ FEA allows for detailed information of the stress data ${ }^{16}$ and also provides the stress in the components of the implant-supported system, ${ }^{2}$ which is not possible through photoelasticity. Based on these particularities of the methodologies, previous studies recommended their combined use. 2,14,16 Despite this recommendation, the reliability between photoelasticity and FEA methodologies are not yet well established for the investigation of the biomechanical behavior of the implant-supported system. Thus, a comparison between these two methodologies under the same simulation is warranted.

Therefore, this study aimed to compare the reliability between photoelasticity and FEA methodologies by evaluating the stresses patterns in conventional and short implants with three different marginal misfit levels. The research hypotheses were as follows: (1) The photoelasticity and FEA methodologies generate similar stress pattern under the same simulation; (2) The increase of marginal misfit is less critical for short and wide implants than conventional implants. 


\section{Materials and Methods}

\section{Study design}

Two conditions were evaluated: 1) model C: two standard Branemark implants (external hexagon $(\mathrm{EH}), 4.1 \times 11 \mathrm{~mm}$; SIN - Sistema de Implante, Sao Paulo, SP, Brazil); and 2) model S: one conventional implant $(\mathrm{EH}, 4.1 \times 11 \mathrm{~mm})$ and one short and wide implant (EH, $5 \times 6$ mm; SIN - Sistema de Implante, Sao Paulo, SP, Brazil), simulating the placement of a short implant in posterior maxilla. Frameworks simulating 3-unit fixed denture prosthesis (FDPs) were obtained by the overcasting method $(n=10)$. Three different levels of marginal misfit were selected based on their misfit average, obtaining three groups: low $(<20 \mu \mathrm{m})$, medium $(>20 \mu \mathrm{m}$ and $<40 \mu \mathrm{m})$ and high (> $40 \mu \mathrm{m})$ misfit. Qualitative photoelastic analysis was used to evaluate the stress level and distribution in periimplant region under two situations: non-loaded (after tightening of frameworks to the models); and 100-N loaded on the molar. Also, FEA was conducted similarly to the photoelasticity design. Maximum and minimum principal strain were recorded numerically and color-coded for the models (non-ductile material) while von Mises stress was obtained for the metallic components (ductile materials) (Figure 1).

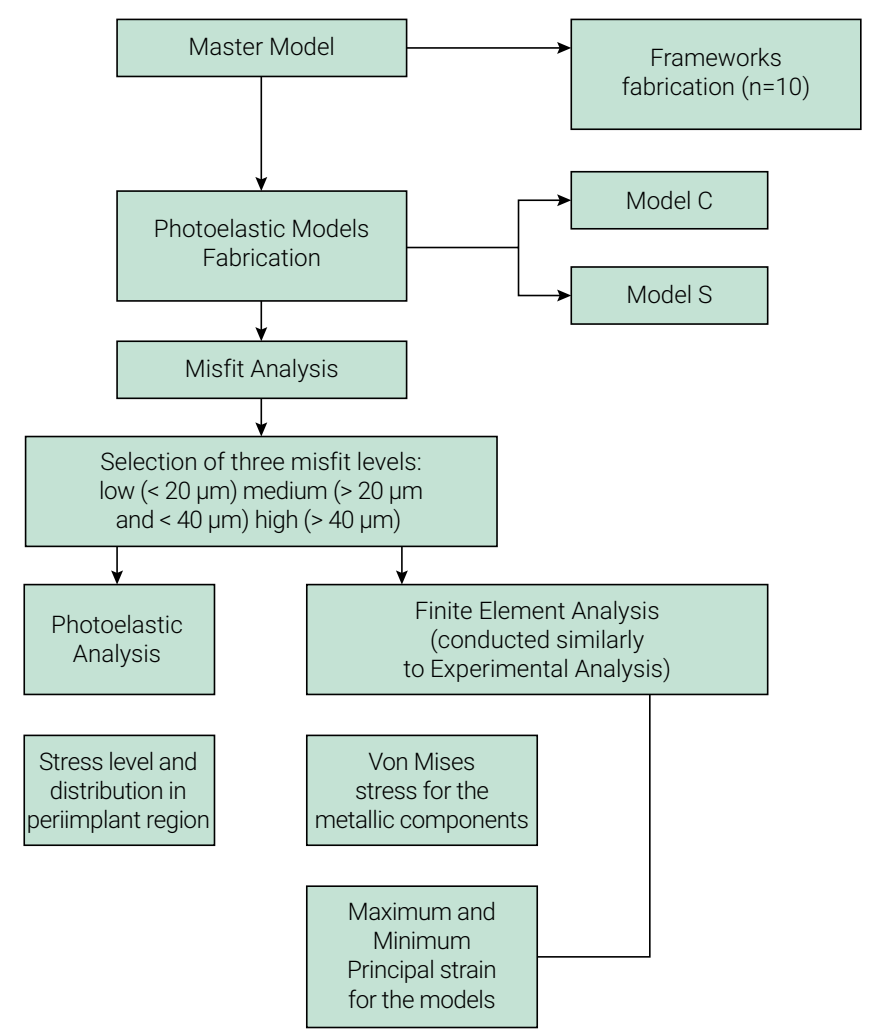

Figure 1. Flowchart of study methodology design. 


\section{Frameworks fabrication}

A steel master model with dimensions of $30 \times 20 \times 15 \mathrm{~mm}$ was fabricated with two drill holes $18 \mathrm{~mm}$ from each other (center to center) and two mini abutment analogs screwed on the model. Overcasted mini abutment cylinders (SIN - Sistema de Implante; Sao Paulo, SP, Brazil) were tightened on the master model. The frameworks simulating FDPs for superior first pre-molar (pillar P) to first molar (pillar M) were waxed with a low-shrinkage acrylic resin (Duralay Il; Reliance Dental Mfg. Co., Chicago, USA). All waxed patterns were sectioned and splinted with a low-shrinkage acrylic resin. The frameworks $(n=10)$ were overcasted in CoCr alloy (Starloy C; Degudent, Dentsply, Hanau-Wolfgang, Hesse, Germany) after including in investment material (Gilvest HS; BK Giulini, Ludwigshafen, Rheinland-Pfalz, Germany). The frameworks were processed by airborne-particle abrasion (110- $\mu \mathrm{m}$ Al2O3 particles under 0.55 MPa air pressure), followed by finishing and polishing with tungsten carbide drills at a low speed, excepted on the metallic strap region.

\section{Photoelastic model fabrication}

A silicone impression (Silibor; Silibor Industria e Comercio Ltd., Sao Paulo, SP, Brazil) was obtained from the master model/transfer set. The implants with mini pillars (Mini Abutment - EH $4.1 \times 2$ mm; SIN - Sistema de Implante, Sao Paulo, SP, Brazil) were tightened on transfers of silicone impression. The photoelastic resin (Araldite GY 279 BR and catalyst Aradur HY 2963; Araltec Chemicals Ltd., Guarulhos, SP, Brazil) was manipulated with the proportion of 2 parts of resin to 1 part of catalyst for 1 minute, leaving a homogeneous mixture. The resin was placed for 20 minutes in a pressure chamber coupled to an air injection tube and at a pressure of $60 \mathrm{kgf} / \mathrm{cm}^{2}$ to prevent bubbles in the material. The photoelastic resin was slowly poured over the impression. The same procedure was performed with short and wide implant and conventional implants to obtain the second photoelastic model. After 72 hours, the transfers were removed from the silicone mold and photoelastic models were obtained for evaluation. Thus, a photoelastic model for each situation was obtained and identified as model $\mathrm{C}$ (conventional) that has two standard Branemark implants, and model $\mathrm{S}$ (short) that has a short and wide implant and a standard Branemark implant.

\section{Marginal misfit evaluation}

The marginal misfit was performed at 120x magnification using a $1.0-\mu \mathrm{m}$ precision microscope (VMM-100-BT; Walter UHL, Asslar, Germany) equipped with a digital camera (KC-512NT; Kodo BR Eletronics Ltd, Sao Paulo, Sao Paulo, Brazil) and analyzer unit (QC 220-HH Quadra-Check 200; Metronics Inc., Bedford, Massachusetts, USA). The procedures also involved a calibrated examiner with an intraclass correlation coefficient of 0.995 ( $p<0.0001$ ), according to the single-screw test protocol, ${ }^{3}$ which shows the marginal misfit reading of the loop while the screw of the opposite pillar is tightened.

The frameworks were positioned on the model and tightened with $10 \mathrm{Ncm}$ using a 0.1-Ncm precision digital torque meter (Torque Meter TQ-8800; - Lutron, Taipei, Taiwan). The readings for pillar $M$ were performed on the buccal and lingual sides in diametrically opposite positions after the pillar P screw was tightened and vice versa. The measurements were performed on both pillars, and an average value of mis- 
fit was obtained for each framework. Three average values of marginal misfit were selected obtaining three groups: low (misfit average $<20 \mu \mathrm{m}$ ); medium (misfit average $>20 \mu \mathrm{m}$ and $<40 \mu \mathrm{m}$ ); and high (misfit average $>40 \mu \mathrm{m}$ ).

\section{Photoelastic analysis}

A horizontal transmission polariscope developed in the Mechanical Design Laboratory Henner Alberto Gomide, School of Mechanical Engineering of Federal University of Uberlandia was used and consisted of two 1/4-retardant wave filters and two polarizing filters, called polarizer and analyzer. A standard position for the photoelastic models was obtained by markings on the polariscope platform. Each framework was tightened to the photoelastic model with 10-Ncm standardized torque, always following the tightening sequence P-M. A layer of mineral oil was applied on the photoelastic models to improve the view of the fringes. The photoelastic models were positioned on the polariscope, and the images were obtained using a digital camera (Canon SX50HS; Miyazaki Daishin Canon Inc., Miyazaki, Japan) in two different conditions: after tightening the frameworks to the photoelastic models, and after applying a load of 100-N on the first molar. In the interval among the analyses, the models were kept under $37^{\circ} \mathrm{C}$ for 10 minutes until no stress was observed using the polariscope, avoiding the presence of residual stress from the previous analysis.

The analysis of each image was performed with a graphic software (Adobe Photoshop CS5 ${ }^{\oplus}$; Adobe Systems, San Jose, CA, USA) according to the visualization of isochromatic fringe order where fringe order of $0=$ black; $1=$ violet/blue transition; and 2, 3, $4=\mathrm{red} /$ green transition..$^{18}$ All images were evaluated by the same operator. The analysis was separated according to the intensity (fringe order) and distribution of stress for different misfit levels and implant designs.

\section{Finite element analysis}

A similar situation of the in vitro assay was modeled and simulated. The framework was scanned by a 3D contact scanner (MODELA MDX-20; Roland, Japan), and the images were imported into the Autodesk Meshmixer 3.0 software (San Diego, CA, USA) to generate the final post-scan image. All metallic components were created based on the real components measurements (VMM-100-BT; Walter UHL, Asslar, Germany; and QC 220HH Quadra-Check 200; Metronics Inc., Bedford, MA, USA) in SolidWorks 2010 (SolidWorks, Concord, MA, USA). Then, six models were assembled based on the misfit level (low, medium and high) and implant design (conventional and short).

The ANSYS Workbench 14 software (Ansys Inc.; Canonsburg, PA, USA) was used to perform the pre-processing, processing and post-processing analyses. All materials were considered as homogeneous, isotropic and elastically linear. The material properties used are shown in Table 1. All contacts were set to frictional $(\mu=0.3),{ }^{17}$ with exception of thread interfaces (screws and implant/araldite), which were set as bonded condition. The meshes were refined in contact areas and checked by element quality (Figure 2), varying from 931.651 to $1,005.189$ nodes and 633.436 to 685.492 elements.

Then, the analysis was divided in two simulations, mechanical loading and molar screw torque. Each simulation was divided in 3 steps, being step 1 and 2 the same for both simulations, which: Step 1: Bolt pre-tension (200-N) at the abutment screws; Step 2: 
Table 1. Materials properties used in the Finite Element Models.

\begin{tabular}{lccc}
\hline Material & Young's modulus $(\mathrm{GPa})$ & Poisson's ratio $(\mathbf{v})$ & Reference \\
\hline Araldite & 2.07 & 0.41 & Anami et al. ${ }^{27}$ \\
\hline Titanium & 110 & 0.28 & Spazzin et al. ${ }^{31}$ \\
\hline CoCr alloy & 185 & 0.35 & Archangelo et. ${ }^{45}$ \\
\hline
\end{tabular}

A

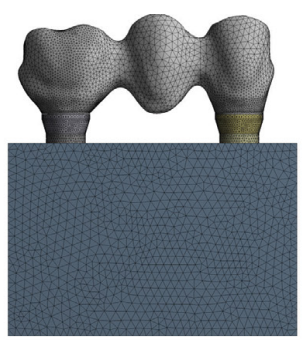

B

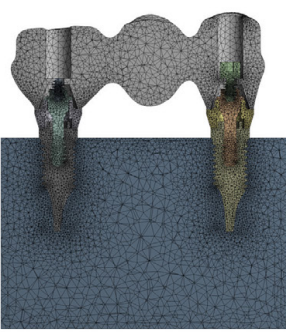

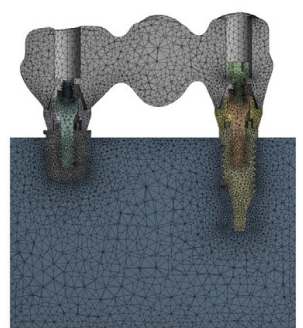

Figure 2. Finite element mesh refined at contact interfaces ( $A$, vestibular view of model; $B$, Model $C$ sectioned; $\mathrm{C}$, Model $\mathrm{S}$ sectioned).

Bolt pre-tension (100-N) at the prosthetic P screw. ${ }^{19}$ Step 3a (M screw torque): The M prosthetic mini screw neck was set to fixed (zero degrees of freedom) and an axial dislodgement (high, medium and low) was applied at molar screw site to simulate misfit sealing by pre-loading. These results were used in comparison to the screw torque test due to simulation limitations with no negatives consequences at obtained results. Step 3b (Mechanical Loading): An axial 100-N force was applied at molar to simulate the masticatory force, and the force opposition was set to araldite basis (zero degress of freedom). Contact prediction was programed between prosthesis and abutment.

The results were obtained after the third step. Maximum and minimum principal strains were obtained for the models, and von Mises stress was obtained for the metallic components.

\section{Results}

\section{In vitro data from photoelastic analysis}

Table 2 and Figure 3 show the stress levels and distribution, respectively, in the models $\mathrm{C}$ and $S$ for different levels of misfit under non-loaded and loaded conditions. The stress intensity is presented according to the higher fringe order observed for each implant. Greater stress level was noted with the increase in the misfit level for both models under nonloaded and loaded conditions (Table 2). After tightening the frameworks, models $\mathrm{C}$ and $\mathrm{S}$ showed similar stress distribution for all situations of misfit. However, peri-implant stress was observed in the full length of the short implant and the same condition is observed after load application (Figure 3). Despite of the load application, both models showed a higher concentration of stress around implant M (Table 2). However, the stresses were dis- 
tributed to implant $\mathrm{P}$ after load application in model $\mathrm{C}$, whereas for model $\mathrm{S}$, the stresses were located almost entirely around the short implant (implant M) (Figure 3).

\section{In silico data from finite element analysis}

Figure 4 and Table 3 show the stress maps distribution and von Mises stress (MPa) levels, respectively, for the metallic components of models $C$ and $S$ for the different levels of misfit. A similar stress maps distribution is observed for all simulated conditions (Figure 4). However, in both models a slight increase of stress is observed in the distocervical region of implant/abutment $P$ as higher is the misfit level. Overall, higher von Mises stress levels in the components is observed for higher misfit levels conditions despite of the implant design (Table 3). The von Mises stress levels of components of pillar $\mathrm{P}$ are not influenced by the presence of short implant (Table 3). Comparing implant $\mathrm{M}$ data, lower von Mises stress values were found for Model S despite of the misfit level (Table 3).

Table 2. Stress intensity (fringe order) according to the model and implant without and with load application for different misfit levels.

\begin{tabular}{lccccccccc}
\hline & \multicolumn{4}{c}{ Model C } & \multicolumn{4}{c}{ Model S } \\
\cline { 2 - 9 } Misfit level & \multicolumn{2}{c}{ No load } & \multicolumn{2}{c}{ With load } & \multicolumn{2}{c}{ No load } & \multicolumn{2}{c}{ With load } \\
\cline { 2 - 9 } & & Implant & Implant & Implant & Implant & Implant & Implant & Implant & Implant \\
& $\mathbf{M}$ & $\mathbf{P}$ & $\mathbf{M}$ & $\mathbf{P}$ & $\mathbf{M}$ & $\mathbf{P}$ & $\mathbf{M}$ & $\mathbf{P}$ \\
\hline Low $(<20 \mu \mathrm{m})$ & 0 & 0 & 5 & 1 & 0 & 0 & 4 & 1 \\
\hline Medium $(>20 \mu \mathrm{m}$ and $<40 \mu \mathrm{m})$ & 1 & 0 & 5 & 2 & 1 & 0 & 5 & 0 \\
\hline High $(>40 \mu \mathrm{m})$ & 3 & 1 & 9 & 4 & 3 & 2 & 7 & 2 \\
\hline
\end{tabular}

* for model S, the implant M represents the short implant.

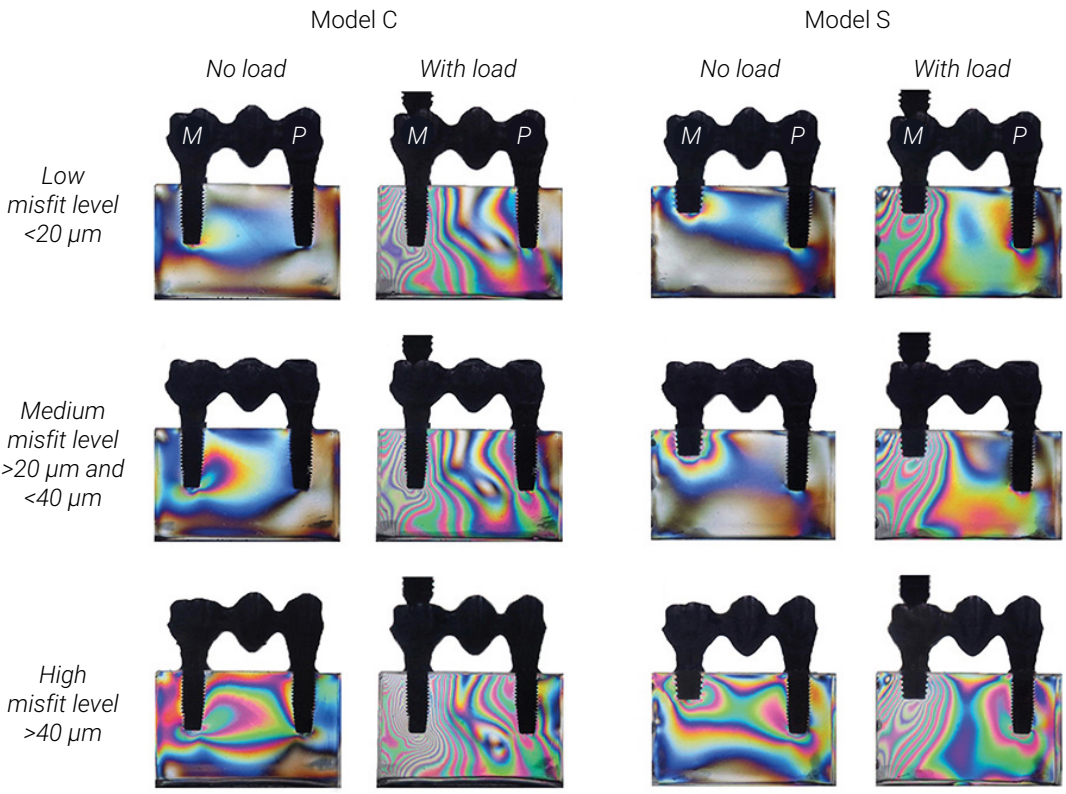

Figure 3. Stress distribution in models $\mathrm{C}$ and $\mathrm{S}$ without load and with load for different misfit levels. 


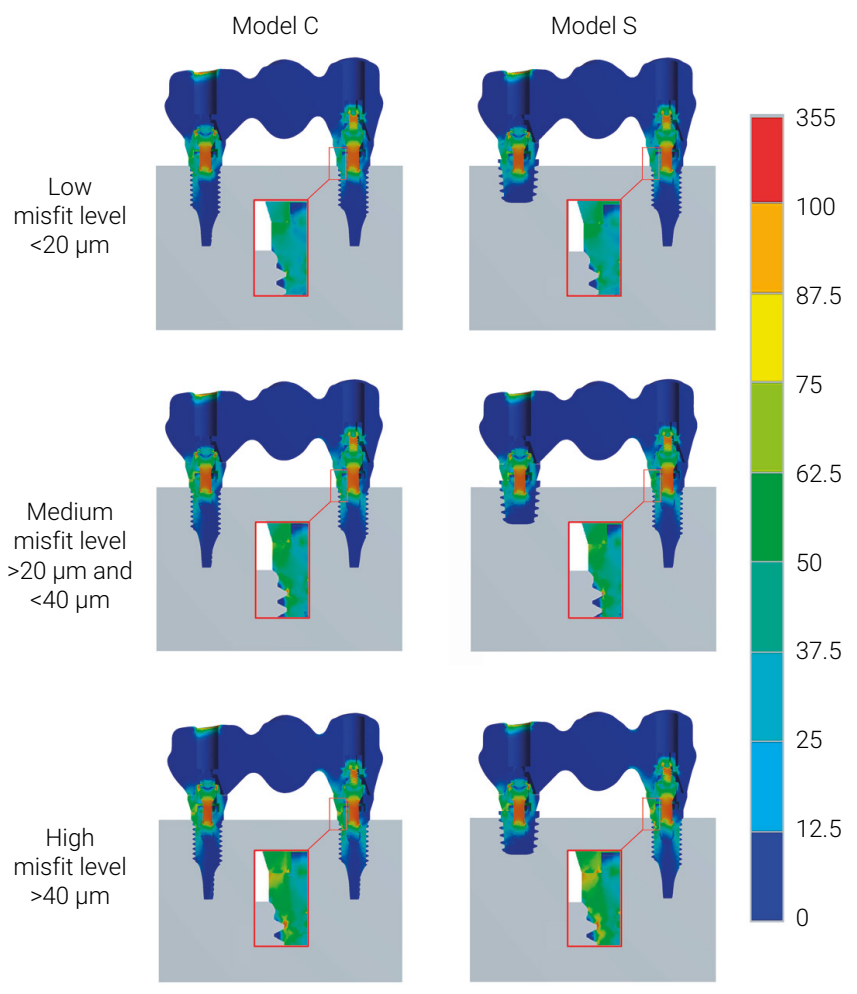

Figure 4. Von Mises stress (MPa) distribution in the metallic components according to the model (model $\mathrm{C}$ and $\mathrm{S}$ ) and the misfit levels (low, medium and high).

Table 3. Maximum von Mises Stress (MPa) levels of the metallic components according to the model for the different misfit levels.

\begin{tabular}{lcccccc}
\hline \multirow{2}{*}{ Component } & \multicolumn{5}{c}{ Misfit level } \\
\cline { 2 - 7 } & \multicolumn{2}{c}{$\begin{array}{c}\text { Low } \\
(<20 \mu \mathrm{m})\end{array}$} & $\begin{array}{c}\text { Medium } \\
(>\mathbf{2 0} \mu \mathrm{m} \text { and }<\mathbf{4 0} \boldsymbol{\mu m})\end{array}$ & $\begin{array}{c}\text { High } \\
(>\mathbf{4 0} \boldsymbol{\mu m})\end{array}$ \\
\cline { 2 - 7 } & Model C & Model S & Model C & Model S & Model C & Model S \\
\hline Framework & 155 & 138 & 158 & 148 & 158 & 159 \\
\hline Abutment P & 78 & 83 & 98 & 100 & 117 & 114 \\
\hline Abutment M & 135 & 128 & 125 & 121 & 134 & 146 \\
\hline Abutment Screw P & 340 & 334 & 339 & 343 & 298 & 306 \\
\hline Abutment Screw M & 354 & 322 & 276 & 279 & 278 & 325 \\
\hline Prosthetic Screw P & 248 & 241 & 238 & 241 & 282 & 279 \\
\hline Prosthetic Screw M* & -- & -- & -- & -- & -- & -- \\
\hline Implant P & 130 & 130 & 134 & 131 & 144 & 147 \\
\hline Implant M & 130 & 105 & 134 & 114 & 139 & 118 \\
\hline Maximum Value & 354 & 334 & 339 & 343 & 298 & 325 \\
\hline * The site of load application eliminates the need of prosthetic screw M component creation for FEA analysis.
\end{tabular}


The $\mu$ strain distribution and levels for models $\mathrm{C}$ and $\mathrm{S}$ for the different misfit levels are shown in figures 5 and 6 . Maximum and minimum principal elastic strain data were selected to illustrate the stress distribution in the resin blocks due to the viewing similarity with the photoelasticity patterns. The $\mu$ strain data was selected to represent the stress levels in the resin block by being the same unity generated by the photoelastic analysis. The higher the misfit level, the greater is the strain concentration (Figure 5). Model S showed higher $\mu$ strain values under all misfit levels simulation when compared to model C (Figure 6). Model S showed higher percentage variation (\%) of von Mises stress values between low and high misfit level (Figure 7).

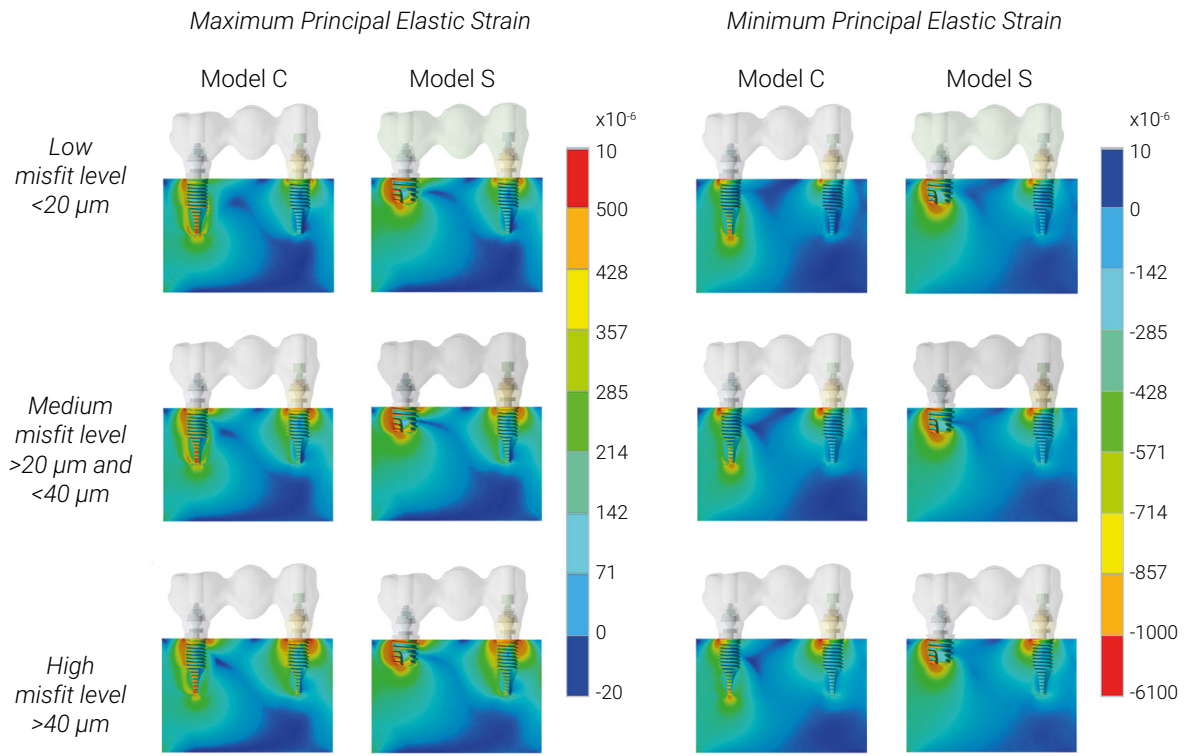

Figure 5. Maximum and Minimum Principal $\mu$ strain distribution of the resin block according the model (model $\mathrm{C}$ and S) and misfit level (low, medium and high).

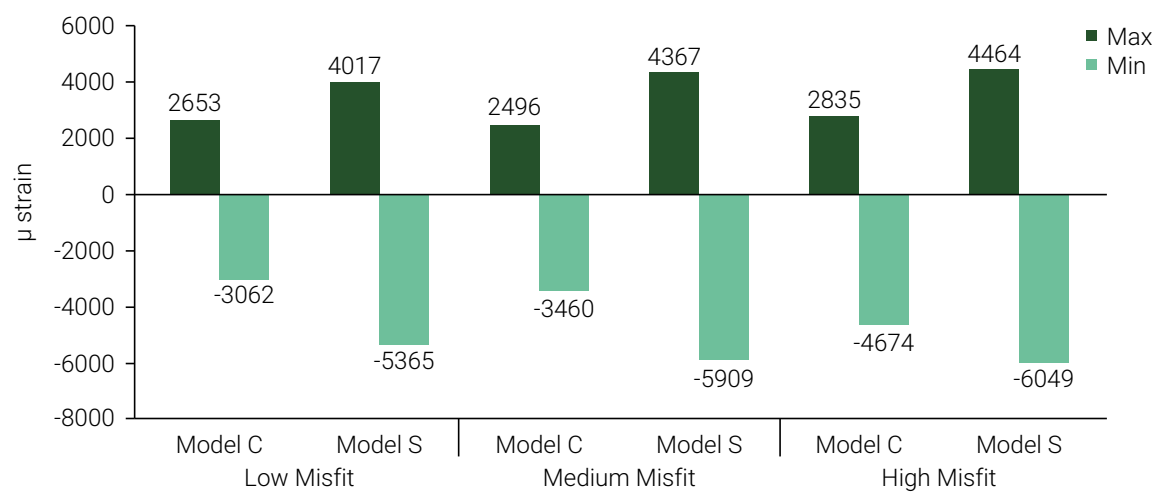

Figure 6. Maximum and Minimum Principal $\mu$ strain levels of the resin block according the model (model $\mathrm{C}$ and $\mathrm{S}$ ) and misfit level (low, medium and high). 


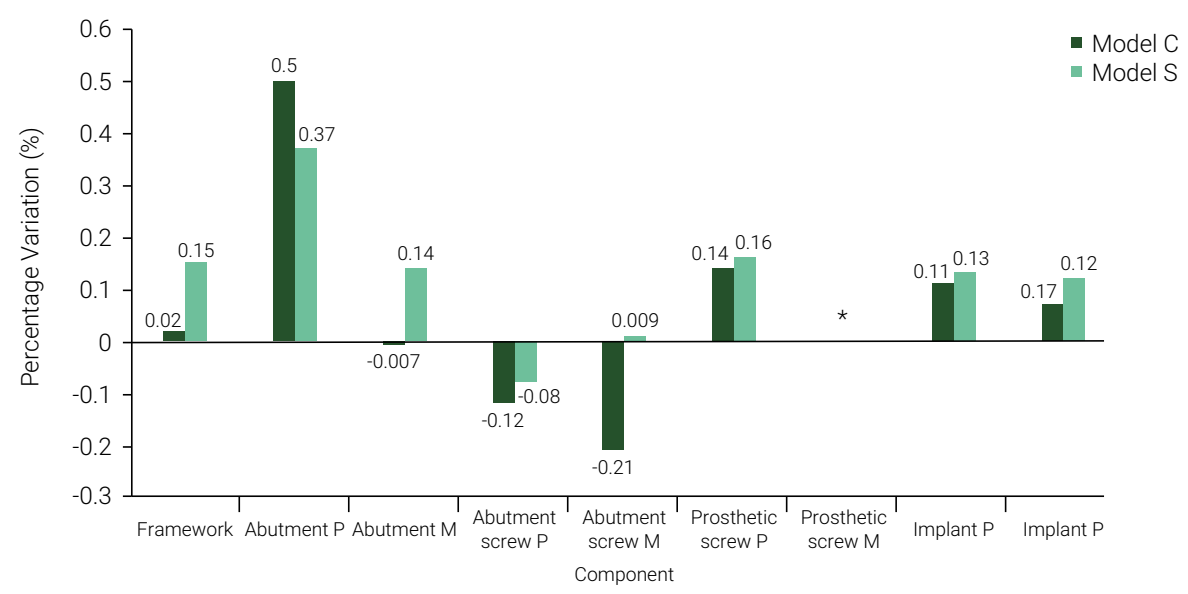

Figure 7. Variation (\%) of von Mises stress values of model $\mathrm{C}$ and $\mathrm{S}$ between low and high misfit level. The reference of total value was the low misfit data. *The prosthetic screw M was not created for FE analysis.

\section{Discussion}

Photoelasticity and FE analyses showed reliable and similar results, accepting the first research hypothesis. Both methodologies detected that the increase of misfit levels generated higher stress levels and produced similar stresses pattern around the implants. Such similar results between photoelasticity and FEA were also reported by a previous study that investigated the stresses generated around implants with different internal-cone abutments. ${ }^{14}$

Photoelasticity is an experimental analysis and allows the use of real components; therefore, the 3D misfit generated by the casting procedure can be accurately reproduced. ${ }^{4}$ In contrast, the reproduction of misfits by FEA is usually simplified by a gap between two components with parallel contact surfaces. ${ }^{2,14}$ Such situation can underestimate the resultant stress levels as noted in the present study. The photoelastic analysis was more sensible to detect the increase of stress with the increase of marginal misfit. However, FEA presents an important advantage providing the stress levels on the components of the system. For these results, a slight difference in the stress concentration is observed among the different misfit levels (Figure 4). Such slight difference can be also justified by the limited simulation of misfit levels by FEA. Thus, the combined use of photoelasticity and FEA is suggested for the investigation of the biomechanical behavior of implant-supported rehabilitations.

In this study, two conditions for the rehabilitation of the posterior maxilla region were simulated. The first condition involved available bone tissue for the placement of two conventional implants. However, in the posterior region the bone tissue is often severely resorbed, requiring surgical procedures such as sinus lift prior to implant placement. The placement of a short implant would avoid these procedures, with promising results in the literature. ${ }^{10,20}$

According to the photoelasticity and FEA results, higher misfit increased the stress levels in the implant-supported system independent of the supporting condition. For 
this study, the condition of total passivity (no misfit) was not evaluated since the absence of a totally passive fit framework is expected. ${ }^{4}$ Although some authors suggest empirical values of misfit between 10 and $150 \mu \mathrm{m}$ to be clinically acceptable, ${ }^{7}$ the findings of this study are that small increments of misfit resulted in an increase of stress intensity and distribution independent of the stress analysis methodology. These findings corroborate that small misfits for screwed implant-supported prosthesis could create a high degree of stress around the implant bodies due to the limited movement (i.e., the absence of periodontal ligament). ${ }^{6}$

The different designs of implants promote differences between the models when compared under the same level of misfit. In contrast with the conventional implant $\mathrm{M}$ of model $\mathrm{C}$, the full length of the short implant in the model $\mathrm{S}$ was involved by stresses areas, observed in both stress analysis. This could be explained by the location of the load; however, this stress concentration pattern is maintained even when the load is not applied in the photoelastic analysis. Although FEA shows stresses in the coronal region of loaded conventional implant (model C) as well (Figure 5), its length allows for a better distribution of stresses levels when compared to the short implant. The presented $\mu$ strain data (Figure 6) confirm the higher concentration of stresses in the full model S. This condition can be potentially damaging for the rehabilitation with short implants. Corroborating these findings, a previous study ${ }^{21}$ concluded that stresses around short and wide implants is increased when compared to conventional implants. Also, the presence of stresses around the coronal third of short implants emphasizes the concern about an increase of bone resorption and risk of failure. ${ }^{22}$ Thus, because bone reabsorption is more critical for short implants due to their length, minimum misfit values should be emphasized for the production of implant fixed prosthesis, ${ }^{5}$ obtaining a condition close to passivity and reducing concentration of stress around coronal region of this implant design.

Concerning the metallic components analysis by FEA, higher von Mises stress values were noted with the increase of misfit level. However, the misfit values simulated in the present study were not sufficient to predict a failure in the components. All the von Mises stress values were lower than the tensile strength for all materials evaluated: $\mathrm{CoCr}$ (552 to $1034 \mathrm{MPa})^{17}$ and $\mathrm{cpTi}$ (480 to $\left.552 \mathrm{MPa}\right)^{23}$ indicating that no failure or mechanical complications would occur under such misfit levels.

Independently of the "safe" von Mises stress values found in components, there are important differences between models $\mathrm{C}$ and $\mathrm{S}$. The presence of the short implant did not affect the von Mises stresses of all components of pillar P (Table 3). However, for the pillar M, lower von Mises stress data was found for the short implant when compared to conventional implant. This can be justified by the higher diameter of the short implant that allows for a better stresses dissipation in the region of the implant/ abutment connection. However, despite of the lower stress values observed in the component, the presence of short implants induced higher $\mu$ strain values on the surrounding system. In addition, higher stress values were detected for almost all components of the model with short implant. This means that the components of model $\mathrm{S}$ showed higher difference on von Mises stress values between the low and high misfit levels. This can suggest that the model $\mathrm{S}$ is more influenced by the misfit increasing. Higher levels of misfit could potentially promote failures for all biomechanical system, 
including in the metallic components.,24 Thus, as the increase of marginal misfit is more critical for short and wide implants for all implant-supported system, the second research hypothesis was denied.

Other types of implant connection and different lengths of short implant were not investigated. Therefore, future studies with different implant connections and lengths are warranted to verify the improvement of biomechanical behavior of short and wide implants. Concerning the stress analysis methodologies, even though photoelasticity and finite element analysis have particularities, there are similarities and important complementary capacity between then. Thus, their combined use may be encouraged for the investigation of the biomechanical behavior of implant-supported rehabilitations.

From this study it can be concluded that the results of photoelasticity and FEA are complementary and present similarities for periimplant evaluation. Also, the increase in marginal misfit produces higher stress concentration in a 3-unit FDP implant-supported rehabilitation. However, it is more critical when a posterior short implant is used.

\section{Acknowledgements}

This study was supported by the Sao Paulo Research Foundation (FAPESP) (grant \#2014/19264-0) and Coordination for the Improvement of Higher Education Personnel (CAPES) (grant \#1778/2014 PROEX). The authors are grateful to Professor Mauro A. A. Nobilo for the polariscope facility.

\section{References}

1. Lindquist LW, Carlsson GE, Jemt T. A prospective 15-year follow-up study of mandibular fixed prostheses supported by osseointegrated implants. Clinical results and marginal bone loss. Clin Oral Implants Res. 1996;7:329-36.

2. Pesqueira AA, Goiato MC, Filho HG, et al. Use of stress analysis methods to evaluate the biomechanics of oral rehabilitation with implants. J Oral Implantol. 2014;40:217-28.

3. Rodrigues SA, Presotto AGC, Barão VAR, et al. The role of welding techniques in the biomechanical behavior of implant-supported prostheses. Mater Sci Eng C. 2017;78:435-42.

4. Presotto AGC, Bhering CLB, Mesquita MF, et al. Marginal fit and photoelastic stress analysis of CADCAM and overcast 3-unit implant-supported frameworks. Journal of Prosthetic Dentistry. 2017;373-9.

5. Spazzin AO, Henriques GEP, de Arruda Nóbilo MA, et al. Influence of prosthetic screw material on joint stability in passive and non-passive implant-supported dentures. Open Dent J. 2009;3:245-9.

6. Watanabe F, Uno I, Hata Y, Neuendorff G, et al. Analysis of stress distribution in a screw-retained implant prosthesis. Int J Oral Maxillofac Implants. 2000;15:209-18.

7. Jemt T. Failures and complications in 391 consecutively inserted fixed prostheses supported by Brånemark implants in edentulous jaws: a study of treatment from the time of prosthesis placement to the first annual checkup. Int J Oral Maxillofac Implants. 1991;6:270-6.

8. Hasan I, Bourauel C, Mundt T, et al. Biomechanics and load resistance of short dental implants: a review of the literature. ISRN Dent. 2013;2013

9. Chang S-H, Lin C-L, Lin Y-S, et al. Biomechanical comparison of a single short and wide implant with monocortical or bicortical engagement in the atrophic posterior maxilla and a long implant in the augmented sinus. Int J Oral Maxillofac Implants. 2012;27:102-11. 
10. Şeker E, Ulusoy M, Ozan O, et al. Biomechanical effects of different fixed partial denture designs planned on bicortically anchored short, graft-supported long, or 45-degree-inclined long implants in the posterior maxilla: a three-dimensional finite element analysis. Int J Oral Maxillofac Implants. 2014;29:1-9.

11. Atieh $\mathrm{M}$ a, Zadeh $\mathrm{H}$, Stanford $\mathrm{CM}$, et al. Survival of short dental implants for treatment of posterior partial edentulism: a systematic review. Int J Oral Maxillofac Implants. 2012;27:1323-31.

12. Isidor F. Loss of osseointegration caused by occlusal load of oral implants. A clinical and radiographic study in monkeys. Vol. 7, Clinical oral implants research. 1996. p. 143-52.

13. Santiago JF, Pellizzer EP, Verri FR, et al. Stress analysis in bone tissue around single implants with different diameters and veneering materials: A 3-D finite element study. Mater Sci Eng C. 2013;33:4700-14.

14. Anami LC, da Costa Lima JM, Takahashi FE, et al. stress distribution around osseointegrated implants with different internal-cone connections: photoelastic and finite element analysis. J Oral Implantol. 2015;41:155-62

15. Kim S, Kim S, Choi H, et al. A three-dimensional finite element analysis of short dental implants in the posterior maxilla. Int J Oral Maxillofac Implant. 2014;29:155-64.

16. Turcio KHL, Goiato MC, Gennari Filho H, et al. Photoelastic analysis of stress distribution in oral rehabilitation. J Craniofac Surg. 2009;20:471-4.

17. Bhering CLB, Bhering, Mesquita MF, Kemmoku DT, et al. Comparison between all-on-four and all-on-six treatment concepts and framework material on stress distribution in atrophic maxilla: A prototyping guided 3D-FEA study. Mater Sci Eng C. 2016;69:715-25.

18. Pereira IP, Consani RLX, Mesquita MF, et al. Photoelastic analysis of stresses transmitted by complete dentures lined with hard or soft liners. Mater Sci Eng C Mater Biol Appl. 2015;55:181-6.

19. Byrne D, Jacobs S, O'Connell B, et al. Preloads generated with repeated tightening in three types of screws used in dental implant assemblies. J Prosthodont. 2006;15:164-71.

20. Monje A, Suarez F, Galindo-Moreno P, et al. A systematic review on marginal bone loss around short dental implants $(<10 \mathrm{~mm})$ for implant-supported fixed prostheses. Clin Oral Implants Res. 2014;25:1119-24.

21. Hasan I, Heinemann F, Aitlahrach M, et al. Biomechanical finite element analysis of small diameter and short dental implant. Biomed Tech (Berl). 2010;55:341-50.

22. Pellizzer EP, de Mello CC, Santiago Junior JF, et al. Analysis of the biomechanical behavior of short implants: The photo-elasticity method. Mater Sci Eng C. 2015;55:187-92.

23. Boyer R, Welsch G and EWC. Materials Properties Handbook: Titanium Alloys. ASM Int Mater Park OH; 1994

24. Spazzin AO, Abreu RT, Noritomi PY, et al. evaluation of stress distribution in overdenture-retaining bar with different levels of vertical misfit. J Prosthodont. 2011;20(4):280-5.

25. Archangelo CM, Rocha EP, Pereira JA, et al. Periodontal ligament influence on the stress distribution in a removable partial denture supported by implant: a finite element analysis. J Appl Oral Sci. 2012;20:362-8. 